\title{
Regiocontrolled Allylic Functionalization of Internal Alkene via Selenium- $\pi$-Acid Catalysis Guided by Boron Substitution
}

\author{
Ling Yang, Yuan Liu, Wen-Xin Fan, Dong-Hang Tan, Qingjiang Li and Honggen Wang* \\ Guangdong Key Laboratory of Chiral Molecule and Drug Discovery, School of Pharmaceutical Sciences, Sun Yat-sen \\ University, Guangzhou 510006, People's Republic of China E-mail: wanghg3@mail.sysu.edu.cn
}

KEYWORDS: alkene, allylic functionalization, selenium, regioselectivity, organoboron

\begin{abstract}
The selenium- $\pi$-acid-catalysis has received increasing attention as powerful tool for olefin functionalization, but the regioselectivity is often problematic. Reported herein is a selenium-catalyzed regiocontrolled olefin transpositional chlorination and imidation reaction. The reaction outcome benefits from an allylic B(MIDA) substitution. And the stabilization of $\alpha$-anion from a hemilabile B(MIDA) moiety was believed to be the key factor for selectivity. Broad substrate scope, good functional group tolerance and generally good yields were observed. The formed products were demonstrated to be valuable precursors for the synthesis a wide variety of structurally complex organoborons.
\end{abstract}

The past few years have witnessed considerable progress in electrophilic chalcogen-catalysis. ${ }^{1}$ In this research realm, the selenium- $\pi$-acid catalysis, ${ }^{2}$ wherein a selenium catalyst chemoselectively activates an alkene or alkyne, is particularly interesting by offering unique reactivities which could be complementary to those of transition metal catalyst ${ }^{3}$ (e.g., gold, ${ }^{4}$ platinum ${ }^{5}$ and palladium ${ }^{6}$ complexes). The high carbophilicity of selenium catalyst offers additional advantages such as good functional group tolerance, simplicity of synthetic operation and mild conditions. However, the utility of selenium- $\pi$-acid catalysis may be plagued by the intrinsically challenging regioselectivity when nonpolarized internal alkenes are used as reaction partners (Scheme 1a). ${ }^{2 \mathrm{c}}$ In a typical reaction manifold, an initial coordination of selenium ion onto olefinic $\pi$-bond leads to the formation of a highly electrophilic seleniranium intermediate A. This intermediate could be stereoselectively, but not regioselectively, trapped by a nucleophile to form two sets of selenide adducts ( $\mathrm{B}$ and $\mathrm{C}$ ). Depending on the reaction parameters (for examples, substituent on the double bond and the oxidant), both adducts could be potentially converted to either allylic, vinylic or 1,2-difunctionalization product. ${ }^{1}$ This was reflected by the seminal work of Sharpless, ${ }^{7}$ wherein the use of unsymmetrical internal alkenes produced the unselective chlorination mixtures (Scheme 1b).

To address the regioselectivity issue, the most commonly used and fruitful strategy is to run the process intramolecularly by introducing a pendent nucleophile to the substrates. ${ }^{8}$ Also effective is the employment of tri-substituted alkenes as substrates, which usually leads to clean tertiary functionalized products as guided by the Markovnikov rule. ${ }^{9}$ A recent study from Zhao suggests that the hydroxyl group resided at the allylic position can function as a directing group (via precoordination with the selenium cation) to enable a selective anti-Markovnikov aza-Wacker imidation reaction (Scheme 1c). ${ }^{10} \mathrm{~A}$ more general solution is by electronic perturbation via the installation of an electron-withdrawing group (EWG) at the allylic position, as firstly reported by Tunge (Scheme $1 \mathrm{~d}$ ). ${ }^{11}$ Thus, by virtue of the reversibility of the chloroselenylation and oxidation processes $\left(\mathbf{A} \rightarrow \mathbf{C}\right.$, Scheme 1a), ${ }^{12}$ a rate-limiting dehydrodeselenenylative elimination eventually leads to an allylic chlor-

SCHEME 1. Selenium- $\pi$-acid catalysis and our work.
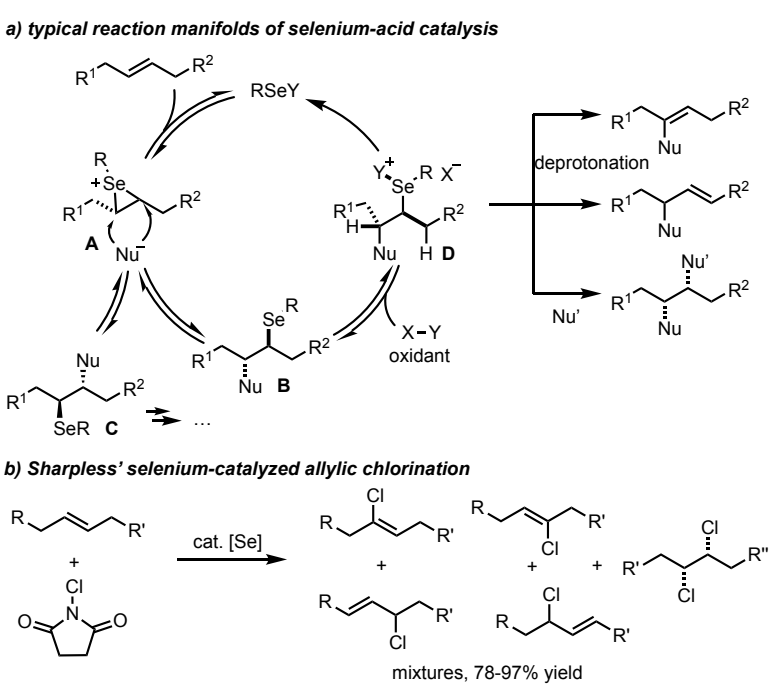

c) Zhao's hydroxyl-directed aza-Wacker amidation reaction.

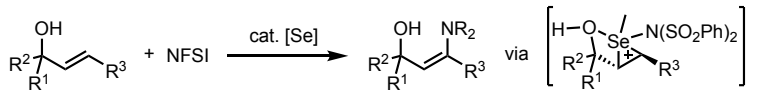

d) EWG-oriented chemo- and regioselective allylic functionalization

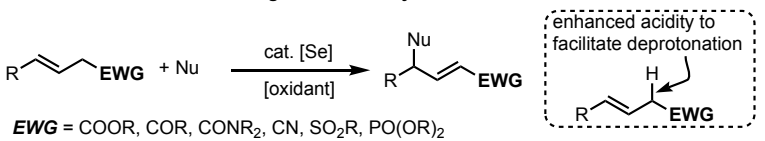

e) this work: boron-enabled allylic chlorination and imidation

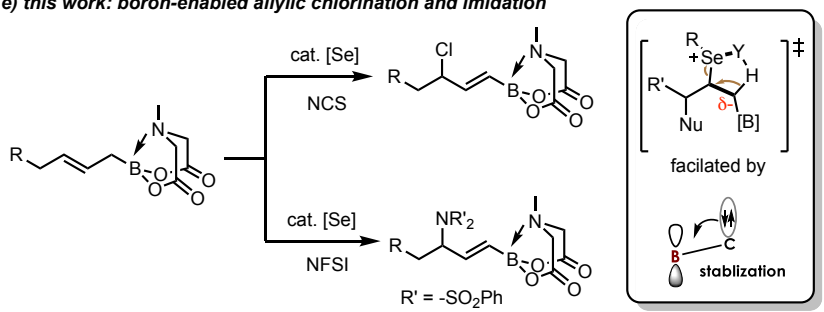

ide product. The acidity of the proton $\alpha$ to the EWG accounts for the profound regioselectivity by facilitating the irreversible deprotonation process. Linear alkenes bearing no allylic EWGs result in complex product mixtures. Similar strategy was later found to be applicable to the elegant selenium- $\pi$-acid catalytic amination, ${ }^{13}$ oxygenation ${ }^{9 b, 14}$ and fluorination ${ }^{9 c, 15}$ reactions. 
On the other hand, the protection of $\mathrm{sp}^{2}$-B organoboron into the pyramidalized $\mathrm{sp}^{3}$-B MIDA ( $N$-methyliminodiacetic acid) boronates brings about tremendously improved stability, which thereby offering new opportunities to the latestage modification of organoborons. ${ }^{16}$ In this context, Previous studies have showcased that in certain cases, the MIDA boron moiety is by no means a bystander, but could confer interesting reactivity to the substrates. ${ }^{17}$ For instance, independent work from Yudin ${ }^{18}$ and us $^{19}$ uncovered an intriguing directing effect of MIDA boron in metal-catalyzed functionalized of alkenes and alkynes, respectively. This effect is attributed to a neighboring stereoelectronic stabilizing donation of electron density to boron, thanks to the hemilabile nature of the MIDA B-N dative bond. ${ }^{20}$ In line with the selenium- $\pi$-acid catalysis, we reason the similar neighboring group effect would offer a chance for a regiocontrolled allylic functionalization reactions. Herein, we report our realization of a selenium-catalyzed double bond transpositional allylic chlorination and imidation of allyl MIDA boronates (Scheme 1e). The reaction leads to the facile synthesis of multifunctional amphoteric building blocks with both the olefin and boron functionality being preserved as valuable handles for further chemical elaboration.
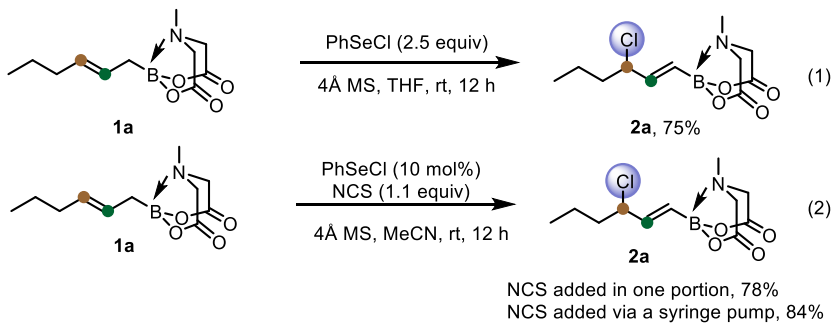

To start, we examined the feasibility of MIDA boron-directed allylic chlorination. The reaction of allyl MIDA boronate $1 \mathrm{a}$ with stoichiometric amount of $\mathrm{PhSeCl}$ as both the oxidant and chlorine source was firstly investigated. A brief survey revealed that mixing $\mathbf{1 a}$ with $\mathrm{PhSeCl}$ ( 2.5 equiv.) in THF in the presence of $4 \AA$ molecular sieve provided cleanly a single allylic chlorination product $\mathbf{2 a}$ in $75 \%$ yield (eq 1 ). The double bond was selectively transpositioned towards the boron moiety and only the $E$ geometric isomer was formed as expected. We also turned our attention to the catalytic version of this reaction. And was found that with 10 mol\% of $\mathrm{PhSeCl}$ catalyst and $N$-chlorosuccinimide (NCS, 1.1 equiv.) in MeCN, a comparable yield of $78 \%$ was obtained. Considering the potential inhibition effect of NCS as observed by Tunge, ${ }^{11}$ the slow addition of NCS via a syringe pump gives an improved yield of $84 \%$ (eq 2).

Encouraged by these results considering the importance of allyl amines, further attempts were also made to explore the applicability of this concept to allylic functionalization imination reactions. With $\mathrm{N}$-fluorobenzenesulfonimide (NFSI, 1.0 equiv.) as oxidant and diphenyl diselane ( $5 \mathrm{~mol} \%$ ) as catalyst in THF at room temperature, an allylic imidation product (25\% yield) was indeed formed with most of the staring materials being untouched (Table 1, entry 1 ). The site and stereoselectivity were in accordance with the above chlorination protocol, confirming the versatility of borondirecting effect. A higher yield was obtained by changing the solvent to MeCN (entries 2 and 3). Further improvements were achieved by introducing molecular sieve (entry 4), increasing the oxidant dosage (entry 5), and slightly raising the reaction temperature (entry 6). And finally, the use of DCE as solvent in associated with $10 \mathrm{~mol} \%$ of catalyst were able to reduce the oxidant (to 1.5 equiv) while enhancing the yield to $68 \%$ (entries 7-9).

\section{TABLE 1. Reaction optimization of allylic imidation. ${ }^{a}$}

\begin{tabular}{ccccccc} 
& & & & & & \\
\hline
\end{tabular}

${ }^{a}$ Reaction condition: $1 \mathrm{a}(0.2 \mathrm{mmol}), \mathrm{NFSI}$ (x equiv.), ( $\mathrm{PhSeSePh}$, y mol\%), $4 \AA \mathrm{MS}(\mathrm{mg})$, temp. $\left({ }^{\circ} \mathrm{C}\right)$, solvent, under $\mathrm{Ar}, 12 \mathrm{~h}$ 。

With the optimized conditions in hand, the substrate scopes for both the oxidation allylic chlorination and imidation reactions were explored. As shown in Scheme 2, a wide variety of alkyl-substituted allyl MIDA boronates were well applicable, generating the $\gamma$-chlorinated/imidized alkenyl boronates both as single regio- and $E$-geometric isomers in moderate to good yields. Some commonly encountered functional groups, such as ester (1f, 1l, 1m), phenoxy (1g), alkoxy (1h), acetal (1) $)$, amide (1j), carbamate (1k), halogen (1n) were all well tolerated, indicating the mildness of the protocols.

\section{SCHEME 2. Substrate Scope.}




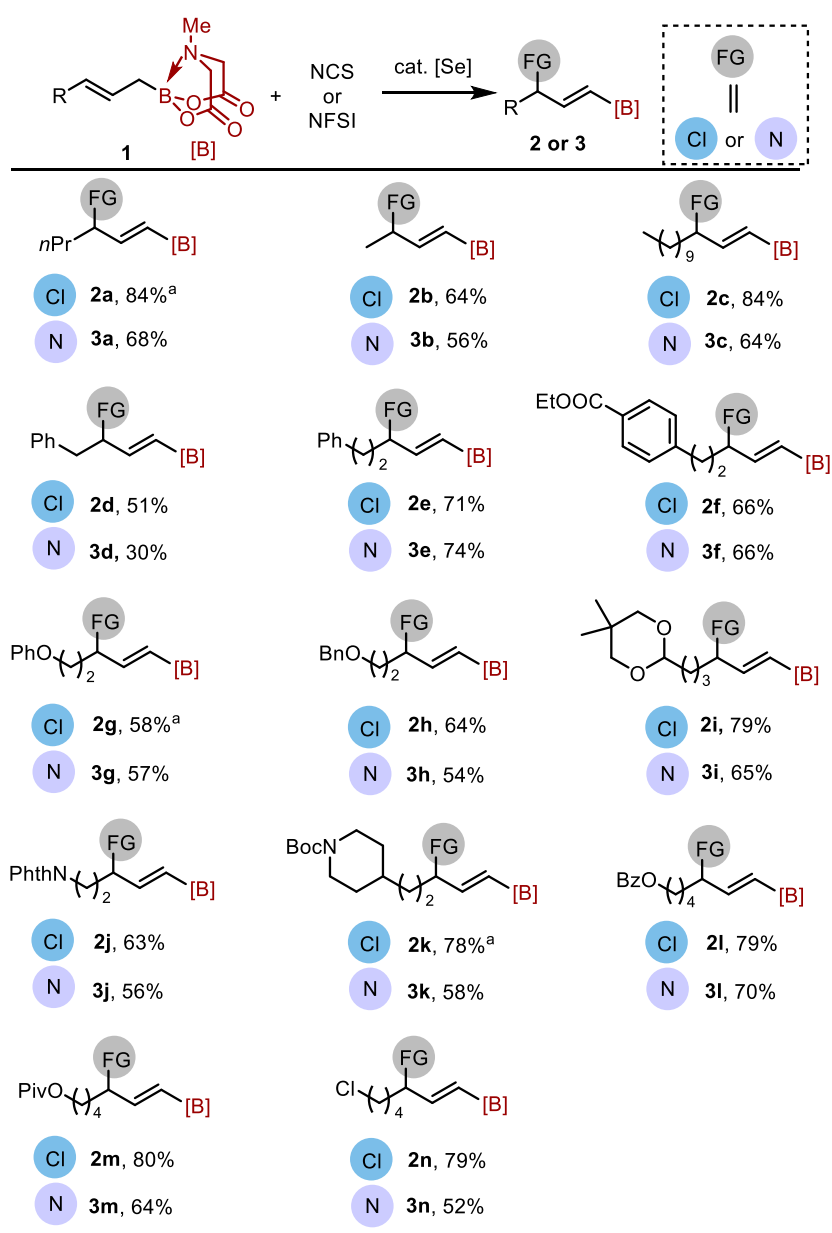

Chlorination: 10 ( $0.2 \mathrm{mmol}), \mathrm{NCS}$ (1.1 equiv.), PhSeCl (10 mol\%), $4 \AA$ MS, MeCN, rt. ${ }^{a}$ NCS (dissolved in MeCN) was added via syringe pump. Imidation: 10 ( $0.2 \mathrm{mmol})$, NFSI (1.5 equiv.), PhSeSePh (10 mol\%), $4 \AA$ MS, DCE, $35^{\circ} \mathrm{C}$, under Ar, $12 \mathrm{~h}$.

Substrate 10 with a $Z$ geometry also successfully delivered the desired chlorination product $\mathbf{2 0}$ with $E$ configuration in good yield (eq 3). However, the imidation product 30 was formed in low yield, probably due to the greater steric hindrance of nitrogen-centered nucleophile.

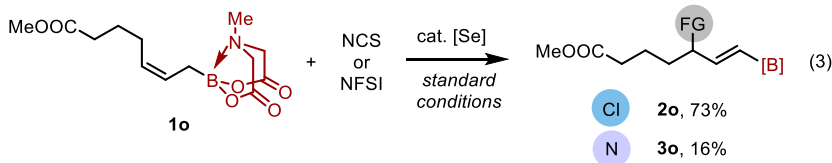

The utility of the formed products was then investigated (Scheme 3). The allylic $\mathrm{C}-\mathrm{Cl}$ bond in $\mathbf{2 a}$ provided valuable handle for follow-up functional group manipulations. For examples, subjecting $\mathbf{2 a}$ to palladium-catalyzed Tsuji-Trost reactions using malonate and sulfinate as nucleophiles delivered the corresponding allylic alkylation (4) and sulfonylation (6) products, respectively, in good yields. A copper-catalyzed displacement of chloride furnished an allyl fluoride 7 without difficulty. ${ }^{21}$ In addition, the iodofluorination of the double bond in 2 a following our previous proto$\operatorname{col}^{22}$ produced the $\alpha$-iodo- $\beta$-fluoro- $\gamma$-chloroalkyl MIDA boronate 8 (confirmed by X-ray single crystal diffraction analysis) ${ }^{23}$ with good regio- and stereoselectivity. And the epoxidation reaction gave efficiently a highly functionalized amphoteric epoxide $\mathbf{9}$.

SCHEME 3. Synthetic derivatizations of $2 a$.

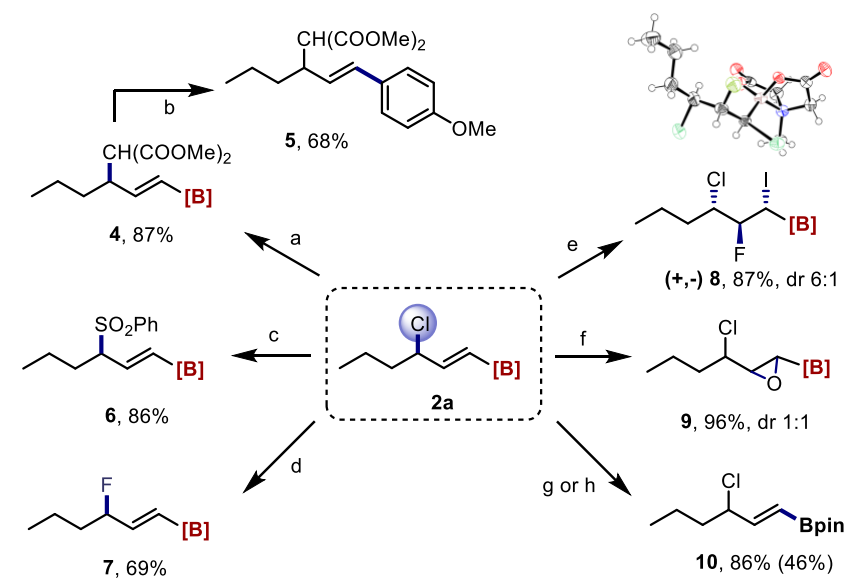

Reaction condition: [a] dimethyl malonate, $\mathrm{NaH}, \mathrm{Pd}(\mathrm{OAc})_{2}$, $\mathrm{PPh}_{3}, \mathrm{THF}, \mathrm{rt}$; [b] $\mathrm{Pd}(\mathrm{OAc})_{2}$, Sphos, $p$-iodoanisole, $1 \mathrm{M} \mathrm{NaOH}$, THF, rt.; [c] sodium benzenesulfinate, $\mathrm{Pd}\left(\mathrm{PPh}_{3}\right)_{4}$, THF/DMSO, $50{ }^{\circ} \mathrm{C}$; [d] AgF, CuBr, MeCN, rt.; [e] DIH, Et $3 \mathrm{~N} \cdot \mathrm{HF}, \mathrm{DCM}, \mathrm{rt}$; [f] mCPBA, DCM, $30{ }^{\circ} \mathrm{C}$; [g] pinacol, $2 \mathrm{M} \mathrm{H}_{2} \mathrm{SO}_{4}$, THF, rt., $1 \mathrm{H}$ NMR yield; [h] isolated yield. For more details, see the Supporting Information. DIH = 1,3-diiodo-5,5-dimethylhydantoin.

Finally, the transesterification reaction of the BMIDA moiety with pinacol allowed the efficient synthesis of alkenyl Bpin 10. And under a slow-release reaction conditions, the palladium-catalyzed Suzuki-Miyaura coupling with aryl iodide was successful to provide the styrenyl product $\mathbf{5}$.

Interestingly, reacting the imidation product $\mathbf{3 a}$ with $\mathrm{NaN}_{3}$ provided the $\gamma$-azidated alkenyl MIDA boronate 11, which can then be applicable to the iodofluorination reaction as well (12). A E2 elimination occurred by heating $\mathbf{3 a}$ with $\mathrm{NaI}$ under microwave irradiation to give a boryl-substituted diene 13.

SCHEME 4. Synthetic derivatizations of $3 a$.

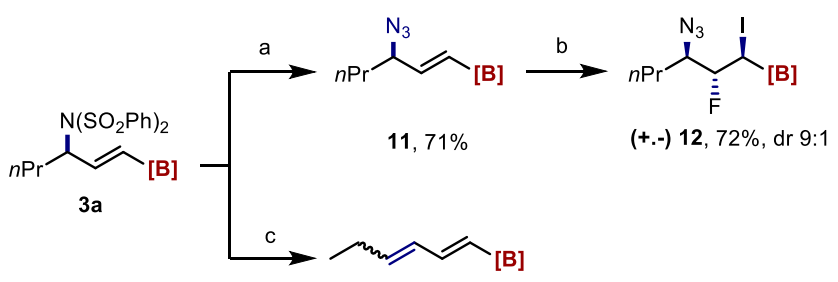

$13,59 \%,(E, E) /(Z, E)=1: 2.5$

Reaction condition: [a] $\mathrm{NaN}_{3}, \mathrm{NaI}, \mathrm{DMF}, 100{ }^{\circ} \mathrm{C}$; [b] DIH, $\mathrm{Et}_{3} \mathrm{~N} \cdot \mathrm{HF}, \mathrm{DCM}, \mathrm{rt}$.; [c] NaI, DMF, $140{ }^{\circ} \mathrm{C}$ (microwave). For more details, see the Supporting Information. DIH = 1,3-diiodo-5,5dimethylhydantoin.

SCHEME 5. Proposed mechanism and mechanistic study. 


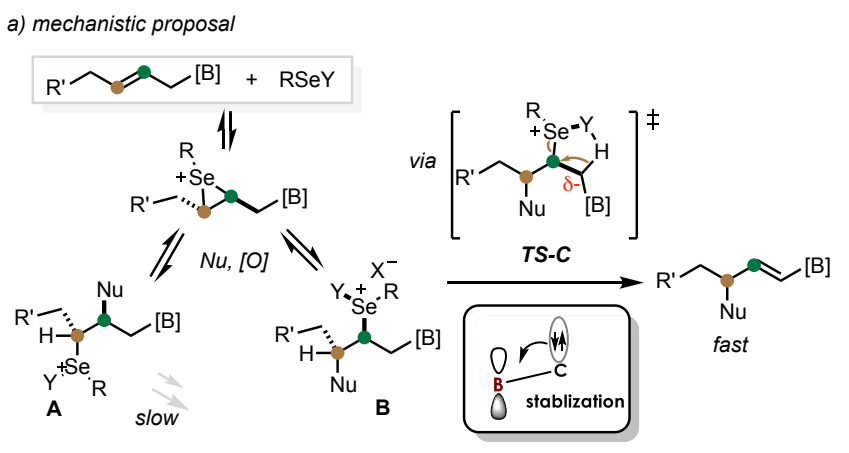

b) kinetic isotope effect study

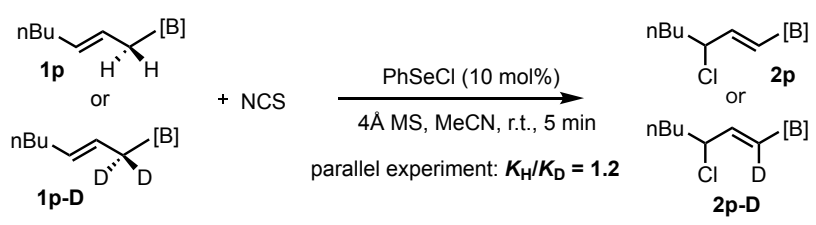

Based on the previous observations on the electron-withdrawing group (EWG)-directed allylic functionalization 11-14, $15 \mathrm{~b}$ and the notion on the capacity of B(MIDA) to accept adjacent electron density, ${ }^{18-20}$ a possible reaction mechanism was proposed (Scheme 5a). The reversible olefin coordination/nucleophilic substitution/selenide oxidation forms two regioisomeric intermediates $\mathbf{A}$ and $\mathbf{B}$. Both are prone to undergo dehydrodeselenenylation or nucleophilic displacement of the selenium moiety. However, the activation effect from B(MIDA) by stabilizing the developing negative charge in the transition state TS-C would facilitate a regioselective syn-dehydrodeselenenylation, thereby shifting the equilibrium towards the alkenyl MIDA boronate formation. Interestingly, previous evidence from Tunge has suggested that the dehydrodeselenenylative elimination is the rate-limiting step. ${ }^{24}$ However, kinetic isotope effect studies in our case by using deuterated allyl MIDA boronate revealed a small KIE value of 1.2 (Scheme $5 b$ ). This result indicates that the $\mathrm{C}-\mathrm{H}$ cleavage is no longer involved in the rate-limiting step, in accordance with an activation effect of B(MIDA) (by lowering the energy of TS-C) in this reaction.

In summary, a selenium- $\pi$-acid-catalyzed regiocontrolled olefin transpositional allylic chlorination and imidation were developed. The reaction outcome benefits from a $B(M I D A)$ substitution. And the stabilization of $\alpha$-anion from a hemilabile B(MIDA) moiety was believed to be the key factor for selectivity, which facilitates the irreversible selenium-mediated syn-H elimination. The protocol led to the synthesis of a wide array of multifunctional amphoteric building blocks with both the olefin and boron functionality being preserved as valuable handles for further chemical elaboration. The utilities of the products were demonstrated. The unique effect of B(MIDA) revealed in this protocol may inspire more interesting boron chemistry from the synthetic community.

\section{ASSOCIATED CONTENT}

Supporting Information.

This material is available free of charge via the Internet at http://pubs.acs.org.
General information, experimental procedures, new compound characterization, crystallographic data, spectroscopic data, and NMR spectra (PDF)

\section{AUTHOR INFORMATION}

\section{Corresponding Author}

Honggen Wang* - Guangdong Key Laboratory of Chiral Molecule and Drug Discovery, School of Pharmaceutical Sciences, Sun Yat-sen University, Guangzhou 510006, People's Republic of China

E-mail: wanghg3@mail.sysu.edu.cn

\section{Authors}

Ling Yang - Guangdong Key Laboratory of Chiral Molecule and Drug Discovery, School of Pharmaceutical Sciences, Sun Yatsen University, Guangzhou 510006, People's Republic of China Yuan Liu - Guangdong Key Laboratory of Chiral Molecule and Drug Discovery, School of Pharmaceutical Sciences, Sun Yatsen University, Guangzhou 510006, People's Republic of China Wen-Xin Fan - Guangdong Key Laboratory of Chiral Molecule and Drug Discovery, School of Pharmaceutical Sciences, Sun Yat-sen University, Guangzhou 510006, People's Republic of China

Dong-Hang Tan - Guangdong Key Laboratory of Chiral Molecule and Drug Discovery, School of Pharmaceutical Sciences, Sun Yat-sen University, Guangzhou 510006, People’s Republic of China

Qingjiang Li - Guangdong Key Laboratory of Chiral Molecule and Drug Discovery, School of Pharmaceutical Sciences, Sun Yat-sen University, Guangzhou 510006, People's Republic of China

\section{Funding Sources}

This work was supported by the National Natural Science Foundation of China (22022114, 21971261), the Guang-dong Basic and Applied Basic Re-search Foundation (2020A1515010624), the Fundamental Research Funds for the Central Universities (20ykzd12) and the Local Innovative and Research Teams Project of Guangdong Pearl River Talents Program (2017BT01Y093).

\section{Notes}

The authors declare no competing interests.

\section{REFERENCES}

(1) Bayeh, L.; Le, P. Q.; Tambar, U. K., Catalytic allylic oxidation of internal alkenes to a multifunctional chiral building block. $\mathrm{Na}$ ture 2017, 547, 196.

(2) (a) Denmark, S. E.; Beutner, G. L., Lewis base catalysis in organic synthesis. Angew. Chem., Int. Ed. 2008, 47, 1560. (b) Breder, A.; Ortgies, S., Recent developments in sulfur- and selenium-catalyzed oxidative and isohypsic functionalization reactions of alkenes. Tetrahedron Lett. 2015, 56, 2843. (c) Ortgies, S.; Breder, A., Oxidative Alkene Functionalizations via Selenium- $\pi$-Acid Catalysis. ACS Catal. 2017, 7, 5828. (d) Guo, R.; Liao, L.; Zhao, X., Electrophilic Selenium Catalysis with Electrophilic N-F Reagents as the Oxidants. Molecules 2017, 22. (e) Rathore, V.; Jose, C.; Kumar, S., Organoselenium small molecules as catalysts for the oxidative functionalization of organic molecules. New J. Chem. 2019, 43, 8852. (f) Shao, L.; Li, Y.; Lu, J.; Jiang, X., Recent progress in selenium-catalyzed organic reactions. Org. Chem. Front. 2019, 6, 2999. (g) Singh, F. V.; Wirth, T., Selenium reagents as catalysts. Catal. Sci. Technol. 2019, 9, 1073. (h) Liao, L.; Zhao, X., Modern Organoselenium Catalysis: Opportunities and Challenges. Synlett 2021, 32, 1262. 
(3) (a) Yamamoto, Y., From sigma- to pi-electrophilic Lewis acids. Application to selective organic transformations. J. Org. Chem. 2007, 72, 7817. (b) Guerra, F.; García-Cabeza, A.; Moreno-Dorado, F.; Ortega, M., Copper-Catalyzed Oxidation of Alkenes and Heterocycles. Synthesis 2016, 48, 2323. (c) Muthusamy, S.; Kumarswamyreddy, N.; Kesavan, V.; Chandrasekaran, S., Recent advances in aerobic oxidation with ruthenium catalysts. Tetrahedron Lett. 2016, 57, 5551.

(4) (a) Corma, A.; Leyva-Perez, A.; Sabater, M. J., Gold-catalyzed carbon-heteroatom bond-forming reactions. Chem. Rev. 2011, 111, 1657. (b) Dorel, R.; Echavarren, A. M., Gold(I)-Catalyzed Activation of Alkynes for the Construction of Molecular Complexity. Chem. Rev. 2015, 115, 9028. (c) Furstner, A., Gold Catalysis for Heterocyclic Chemistry: A Representative Case Study on Pyrone Natural Products. Angew. Chem., Int. Ed. 2018, 57, 4215.

(5) Furstner, A.; Davies, P. W., Catalytic carbophilic activation: catalysis by platinum and gold pi acids. Angew. Chem., Int. Ed.2007, $46,3410$.

(6) (a) Moiseev, I. I.; Vargaftik, M. N., Allylic oxidation of alkenes with palladium catalysts. Coord. Chem. Rev. 2004, 248, 2381. (b) McDonald, R. I.; Liu, G.; Stahl, S. S., Palladium(II)-catalyzed alkene functionalization via nucleopalladation: stereochemical pathways and enantioselective catalytic applications. Chem. Rev. 2011, 111, 2981. (c) Yin, G.; Mu, X.; Liu, G., Palladium(II)-Catalyzed Oxidative Difunctionalization of Alkenes: Bond Forming at a High-Valent Palladium Center. Acc. Chem. Res. 2016, 49, 2413.

(7) (a) Hori, T.; Sharpless, K. B., Selenium-catalyzed nonradical chlorination of olefins with $\mathrm{N}$-chlorosuccinimide. J. Org. Chem. 2002, 44, 4204. (b) Hori, T.; Sharpless, K. B., Conversion of allylic phenylselenides to the rearranged allylic chlorides by $\mathrm{N}$-chlorosuccinimide. Mechanism of selenium-catalyzed allylic chlorination of beta-pinene. J. Org. Chem. 2002, 44, 4208.

(8) (a) Browne, D. M.; Niyomura, O.; Wirth, T., Catalytic use of selenium electrophiles in cyclizations. Org. Lett. 2007, 9, 3169. (b) Singh, F. V.; Wirth, T., Selenium-catalyzed regioselective cyclization of unsaturated carboxylic acids using hypervalent iodine oxidants. Org. Lett. 2011, 13, 6504. (c) Guo, R.; Huang, J.; Huang, H.; Zhao, X., Organoselenium-Catalyzed Synthesis of Oxygen- and NitrogenContaining Heterocycles. Org. Lett. 2016, 18, 504. (d) Kawamata, Y.; Hashimoto, T.; Maruoka, K., A Chiral Electrophilic Selenium Catalyst for Highly Enantioselective Oxidative Cyclization. J. Am. Chem. Soc. 2016, 138, 5206. (e) Ortgies, S.; Rieger, R.; Rode, K.; Koszinowski, K.; Kind, J.; Thiele, C. M.; Rehbein, J.; Breder, A., Mechanistic and Synthetic Investigations on the Dual Selenium- $\pi$-Acid/Photoredox Catalysis in the Context of the Aerobic Dehydrogenative Lactonization of Alkenoic Acids. ACS Catal. 2017, 7, 7578. (f) Breder, A.; Rode, K.; Palomba, M.; Ortgies, S.; Rieger, R., Aerobic Allylation of Alcohols with Non-Activated Alkenes Enabled by Light-Driven Selenium- $\pi-$ Acid Catalysis. Synthesis 2018, 50, 3875.

(9) (a) Raucher, S., The synthesis of vinyl bromides and allyl bromides from monosubstituted alkenes. Tetrahedron Lett. 1977, 18, 3909. (b) Torii, S.; Uneyama, K.; Ono, M.; Bannou, T., Generation and recycle use of selenenylating reagents in electrochemical oxyselenenylation-deselenenylation of olefins. J. Am. Chem. Soc. 1981, 103, 4606. (c) Bloom, S.; Knippel, J. L.; Holl, M. G.; Barber, R.; Lectka, T., A cooperative allylic fluorination: combination of nucleophilic and electrophilic fluorine sources. Tetrahedron Lett. 2014, 55, 4576.

(10) Deng, Z.; Wei, J.; Liao, L.; Huang, H.; Zhao, X., Organoselenium-catalyzed, hydroxy-controlled regio- and stereoselective amination of terminal alkenes: efficient synthesis of 3-amino allylic alcohols. Org. Lett. 2015, 17, 1834.

(11) Tunge, J. A.; Mellegaard, S. R., Selective selenocatalytic allylic chlorination. Org. Lett. 2004, 6, 1205.

(12) (a) Clive, D. L. J.; Russell, C. G.; Chittattu, G.; Singh, A., Cyclofunctionalisation of unsaturated acids with benzeneselenenyl chloride. Tetrahedron 1980, 36, 1399. (b) Denmark, S. E.; Edwards, M.
G., On the mechanism of the selenolactonization reaction with selenenyl halides. J. Org. Chem. 2006, 71, 7293.

(13) Trenner, J.; Depken, C.; Weber, T.; Breder, A., Direct oxidative allylic and vinylic amination of alkenes through selenium catalysis. Angew. Chem., Int. Ed. 2013, 52, 8952.

(14) (a) Tiecco, M.; Testaferri, L.; Tingoli, M.; Bagnoli, L.; Santi, C., Catalytic conversion of $(\beta, \gamma$-unsaturated esters, amides and nitriles into $\gamma$-alkoxy or $\gamma$-hydroxy $\alpha, \beta$-unsaturated derivatives induced by persulfate anion oxidation of diphenyl diselenide. J. Chem. Soc., Chem. Commun. 1993, 637. (b) Ortgies, S.; Depken, C.; Breder, A., Oxidative Allylic Esterification of Alkenes by Cooperative Selenium-Catalysis Using Air as the Sole Oxidant. Org. Lett. 2016, 18, 2856. (c) Depken, C.; Kratzschmar, F.; Rieger, R.; Rode, K.; Breder, A., Photocatalytic Aerobic Phosphatation of Alkenes. Angew. Chem., Int. Ed. 2018, 57, 2459.

(15) (a) Uneyama, K.; Asai, H.; Dan-oh, Y.; Matta, H., Electrochemical generation of [PhSeF] for fluoro-selenation of alkenes and alkynes and its recycle use. Electrochim. Acta 1997, 42, 2005. (b) Guo, R.; Huang, J.; Zhao, X., Organoselenium-Catalyzed Oxidative Allylic Fluorination with Electrophilic N-F Reagent. ACS Catal. 2018, 8, 926.

(16) (a) Mancilla, T.; Contreras, R.; Wrackmeyer, B., New bicyclic organylboronic esters derived from iminodiacetic acids. Journal of Organometallic Chemistry 1986, 307, 1. (b) He, Z.; Yudin, A. K., Amphoteric alpha-boryl aldehydes. J. Am. Chem. Soc. 2011, 133, 13770. (c) Li, J.; Burke, M. D., Pinene-derived iminodiacetic acid (PIDA): a powerful ligand for stereoselective synthesis and iterative crosscoupling of C(sp3) boronate building blocks. J. Am. Chem. Soc. 2011, 133, 13774. (d) He, Z.; Trinchera, P.; Adachi, S.; St Denis, J. D.; Yudin, A. K., Oxidative geminal functionalization of organoboron compounds. Angew. Chem., Int. Ed. 2012, 51, 11092. (e) Xu, L.; Ding, S.; $\mathrm{Li}, \mathrm{P}$., Site-differentiated polyboron arenes prepared by direct C-H borylation and their highly selective Suzuki-Miyaura cross-coupling reactions. Angew. Chem., Int. Ed. 2014, 53, 1822. (f) Li, J.; Ballmer, S. G.; Gillis, E. P.; Fujii, S.; Schmidt, M. J.; Palazzolo, A. M.; Lehmann, J. W.; Morehouse, G. F.; Burke, M. D., Synthesis of many different types of organic small molecules using one automated process. Science 2015, 347, 1221. (g) Li, J.; Grillo, A. S.; Burke, M. D., From synthesis to function via iterative assembly of N-methyliminodiacetic acid boronate building blocks. Acc. Chem. Res. 2015, 48, 2297. (h) Taguchi, J.; Ikeda, T.; Takahashi, R.; Sasaki, I.; Ogasawara, Y.; Dairi, T.; Kato, N.; Yamamoto, Y.; Bode, J. W.; Ito, H., Synthesis of Acylborons by Ozonolysis of Alkenylboronates: Preparation of an Enantioenriched Amino Acid Acylboronate. Angew. Chem., Int. Ed. 2017, 56, 13847;

(17) (a) Zeng, Y. F.; Ji, W. W.; Lv, W. X.; Chen, Y.; Tan, D. H.; Li, Q.; Wang, H., Stereoselective Direct Chlorination of Alkenyl MIDA Boronates: Divergent Synthesis of $\mathrm{E}$ and $\mathrm{Z}$ alpha-Chloroalkenyl Boronates. Angew. Chem., Int. Ed. 2017, 56, 14707. (b) Lee, C. F.; Diaz, D. B.; Holownia, A.; Kaldas, S. J.; Liew, S. K.; Garrett, G. E.; Dudding, T.; Yudin, A. K., Amine hemilability enables boron to mechanistically resemble either hydride or proton. Nat. Chem. 2018, 10, 1062. (c) Lv, W. X.; Li, Q.; Li, J. L.; Li, Z.; Lin, E.; Tan, D. H.; Cai, Y. H.; Fan, W. X.; Wang, H., gem-Difluorination of Alkenyl N-methyliminodiacetyl Boronates: Synthesis of alpha- and beta-Difluorinated Alkylborons. Angew. Chem., Int. Ed. 2018, 57, 16544. (d) Tan, D. H.; Cai, Y. H.; Zeng, Y. F.; Lv, W. X.; Yang, L.; Li, Q.; Wang, H., DiversityOriented Synthesis of alpha-Functionalized Acylborons and Borylated Heteroarenes by Nucleophilic Ring Opening of alphaChloroepoxyboronates. Angew. Chem., Int. Ed. 2019, 58, 13784. (e) Zeng, Y. F.; Liu, X. G.; Tan, D. H.; Fan, W. X.; Li, Y. N.; Guo, Y.; Wang, H., Halohydroxylation of alkenyl MIDA boronates: switchable stereoselectivity induced by $\mathrm{B}(\mathrm{MIDA})$ substituent. Chem. Commun. 2020, 56, 4332. (f) Yang, L.; Tan, D. H.; Fan, W. X.; Liu, X. G.; Wu, J. Q.; Huang, Z. S.; Li, Q.; Wang, H., Photochemical Radical C-H Halogenation of Benzyl N-Methyliminodiacetyl (MIDA) Boronates: 
Synthesis of alpha-Functionalized Alkyl Boronates. Angew. Chem., Int. Ed. 2021, 60, 3454

(18) Corless, V. B.; Holownia, A.; Foy, H.; Mendoza-Sanchez, R.; Adachi, S.; Dudding, T.; Yudin, A. K., Synthesis of alpha-Borylated Ketones by Regioselective Wacker Oxidation of Alkenylboronates. Org. Lett. 2018, 20, 5300.

(19) Lin, E. E.; Wu, J.-Q.; Schäfers, F.; Su, X.-X.; Wang, K.-F.; Li, J.L.; Chen, Y.; Zhao, X.; Ti, H.; Li, Q.; Ou, T.-M.; Glorius, F.; Wang, H., Regio- and stereoselective synthesis of tetra- and triarylethenes by $\mathrm{N}$-methylimidodiacetyl boron-directed palladium-catalysed threecomponent coupling. Commun. Chem. 2019, 2, 34.

(20) Liu, Y.; Luo, W.; Wu, J.; Fang, Y.; Li, Y.; Jin, X.; Zhang, L.; Zhang, Z.; Xu, F.; Du, C., Radical addition-polar termination cascade: efficient strategy for photoredox-neutral-catalysed cyclopropanation and Giese-type reactions of alkenyl N-methyliminodiacetyl boronates. Org. Chem. Front. 2020, 7, 1588.

(21) Zhang, Z.; Wang, F.; Mu, X.; Chen, P.; Liu, G., Copper-catalyzed regioselective fluorination of allylic halides. Angew. Chem., Int. Ed. 2013, 52, 7549.

(22) Fan, W. X.; Li, J. L.; Lv, W. X.; Yang, L.; Li, Q.; Wang, H., Synthesis of fluorinated amphoteric organoborons via iodofluorination of alkynyl and alkenyl MIDA boronates. Chem. Commun. 2020, $56,82$.

(23) See the Supporting Information (CCDC: 2077463).

(24) Mellegaard-Waetzig, S. R.; Wang, C.; Tunge, J. A., Seleniumcatalyzed oxidative halogenation. Tetrahedron 2006, 62, 7191. 
